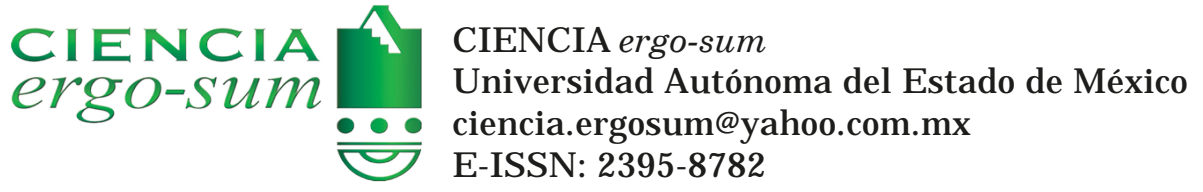

\title{
Conectivismo y neuroeducación: transdisciplinas para la formación en la era digital
}

Islas Torres, Claudia

Conectivismo y neuroeducación: transdisciplinas para la formación en la era digital

CIENCIA ergo-sum, vol. 28, núm. 1, marzo-junio 2021 |e117

Universidad Autónoma del Estado de México, México

Esta obra está bajo una Licencia Creative Commons Atribución-NoComercial-SinDerivar 4.0 Internacional .

Islas Torres, C. (2021). Conectivismo y neuroeducación: transdisciplinas para la formación en la era digital. CIENCIA ergo-sum, 28(1). http://doi.org/10.30878/ces.v28n1all 


\title{
Conectivismo y neuroeducación: transdisciplinas para la formación en la era digital
}

\section{Connectivism and Neuroeducation: Trans-disciplines for teaching in the digital era}

\author{
Claudia Islas Torres \\ Universidad de Guadalajara, México \\ cislas@cualtos.udg.mx \\ (D) https://orcid.org/0000-0001-9929-4990
}

Recepción: 2 de diciembre de 2019

Aprobación: 16 de junio de 2020

\begin{abstract}
RESUMEN
Se hace una revisión de la literatura con el objetivo exponer crítica y analíticamente cómo el conectivismo y la neuroeducación son transdisciplinas que explican procesos formativos en la era digital. La información develó dinámicas e interpretaciones desde diferentes posturas epistémicas. En la fase heurística se tomaron criterios como año de publicación, idioma, metodología, hallazgos; se procedió a la fase hermenéutica donde se interpretó y concluyó que la era tecnocientífica ha traído consecuencias y cambios en los sistemas de enseñanza-aprendizaje, por lo que se requieren referentes que adviertan cómo es el aprendizaje y la construcción de conocimiento no necesariamente en la mente sino a través de dispositivos e infraestructuras tecnológicas que obligan también a la comprensión del funcionamiento del cerebro.
\end{abstract}

Palabras Clave: conectivismo, neuroeducación, proceso de formación, enseñanza-aprendizaje, transdisciplina.

\begin{abstract}
The review of the literature presented was goal critically and analytically exposing how connectivism and neuroeducation are transdisciplines that explain formative processes in the digital age. The information revealed dynamics, and interpretations of these, from different epistemic positions. In the heuristic phase criteria were taken such as: year of publication, language, methodology, findings. We proceeded to the hermeneutic phase where it was interpreted and concluded that the techno-scientific era has brought consequences and changes to the teaching-learning systems, so references are required that warn what learning and knowledge construction are not necessarily in the mind, but through devices and technological infrastructures, also forcing the understanding of the the brain's functioning.
\end{abstract}

KeYwORDs: Conectivism, Neuroeducation, Teaching process, Teaching-Learning, Trans-discipline

\section{INTRODUCCIÓN}

Los humanos nacemos con el instinto de aprender y adquirir nuevos conocimientos sobre el entorno, por lo que se requiere de atención para que lo aprendido adquiera significado y sentido (García, 2019); sin embargo, en la actualidad se dispone de demasiada información para la toma de decisiones o para mantenerse enterado sobre determinado tema. La mayoría de las personas en el mundo se encuentran conectadas, lo que eventualmente conduce a que se consideren poseedoras de todo el conocimiento al instante sin darse cuenta que éste cambia o se transforma y se configura como algo distinto a lo que en un principio fue creado; una verdad que hoy pudiera ser absoluta en diez años ya no lo será (Bueno, 2017; Cabero y Llorente, 2015).

En este sentido, la información se actualiza de manera constante y contradice los conocimientos con los que ya se contaba. Con ello, tanto cambio tecnológico y social deja a las personas desconectadas, desorientadas o aburridas (García, 2019). 
Es notorio que con el impacto de los medios digitales mayor es el número de procesos y entornos que intervienen e influyen en la generación del conocimiento; por ende, la era de la sociedad-red requiere de concepciones más flexibles de éste para que el discente sea protagonista activo. Así, "aprender a aprender" es imprescindible para que se tenga un aprendizaje vivencial y obtener de esta manera el mejor provecho de sus experiencias; por lo anterior, se podría decir que el aprendizaje y la construcción de conocimiento es un proceso integrado de adaptación continuo para que el cerebro de los individuos sea comprendido en cuanto a la manera en que funciona y actúa para generar aprendizajes (Bueno, 2017).

En consecuencia, el docente adquiere la responsabilidad de guiar y enseñar a pensar por medio de la metacognición (proceso en el que el alumno desarrolla la capacidad de reflexionar, comprender y regular su aprendizaje) beneficiándose en su autogestión, puesto que pone en marcha procesos cognitivos que regularmente no habilita (Guerrero, 2019). Por ello, el docente tratará de conseguir que cada alumno saque el máximo provecho individual y colectivo de sus habilidades y capacidades dentro de la diversidad que se presenta en las aulas (Bueno, 2017).

Partiendo de lo anterior, se opina que la educación repercute en el cerebro de los aprendices y de los docentes porque el proceso es bidireccional y, al mismo tiempo que se modifican las conexiones en los cerebros de los alumnos, también sus acciones inciden sobre el cerebro de los docentes (Sitti, Sopeerak, y Sompong, 2013). Aunado a esto, es importante resaltar que la vigencia de contenidos que ya eran conocidos por estos actores aún lo siguen siendo, aunque las maneras de llegar a ellos sean diferentes; por eso es necesario reflexionar respecto a la imperante transformación y actualización de las pedagogías que se practican para basarlas en evidencias profesionales que sean indicios del éxito o fracaso de implementar estrategias o herramientas que parecieran estar de moda o ser las privilegiadas del momento.

Asimismo, es necesario reconocer que los cambios dados por la incursión de las tecnologías en los procesos formativos requieren de disciplinas que acerquen a la comprensión del aprendizaje en una era revolucionada por el mundo digital. En este sentido, la distribución caótica de la información hace necesario adaptar las estrategias de aprendizaje y enseñanza a nuevos mecanismos de pensamiento y a las dinámicas no lineales de la red.

Por lo anterior, se supondría que la educación debe generar modelos de enseñanza que impliquen la incorporación de medios digitales; en este caso, no se trata sólo de enseñar contenidos, sino de cómo enseñar procesos para que a la luz del nuevo conocimiento se comprenda también el funcionamiento del cerebro respecto a cómo atiende, aprende, memoriza y soluciona problemas (Ramón, 2015) desde el acceso a contenidos en formatos diferentes que modifican la linealidad con la que se esta acostumbrado a aprender.

En este sentido, la información recopilada para este artículo pretende exponer desde una perspectiva analíticocrítica la manera en el que el conectivismo y la neuroeducación pueden ser transdisciplinas que se pueden emplear como soporte de los procesos formativos en la era digital y que al mismo tiempo expliquen el suceder del aprendizaje en el siglo XXI.

\section{Conectivismo, ¿QUÉ es?}

Hace varias décadas los investigadores de la educación se han preocupado por entender los procesos de aprendizaje desde distintas vertientes y posturas, lo cual ha originado que exista un elevado porcentaje de literatura que intenta dar a conocer cómo sucede o puede ser el aprendizaje en el siglo XXI. Si bien es cierto que cada vez existe más información al respecto, también se reconoce que no hay elementos empíricos suficientes que, gracias a datos comprobables, den soporte a este cúmulo de conocimientos que ha surgido y dejan en duda las aseveraciones que distintos autores exponen, como es el caso del conectivismo.

Hay quienes reconocen el conectivismo como una teoría de aprendizaje (Downes, 2012; Siemens, 2005; Sánchez-Cabrera et al., 2019; Sagar, 2014), mientras que otros insisten en que no cuenta con los elementos necesarios comprobables para considerarla como tal (Cabero y Llorente, 2015; Padrón, 2014; Czerwonogora, 2014; Sagar, 2014). 
A partir de lo anterior, para comprender la implicación del conectivismo en los procesos formativos es necesario tener claridad sobre la conceptualización del aprendizaje, que se ha definido desde diferentes teorías y enfoques epistémicos; por ejemplo, el conductismo lo concebía como la reproducción de lo enseñado por medio de estructuras de estímulos o información diseñada por el instructor hasta llegar al punto de que no se requería la actividad mental de los alumnos; la repetición se había mecanizado de tal manera que el estudiante emitía los resultados esperados (Pozo y Monereo, 2013).

Sin embargo, este tipo de conceptualización no fue suficiente, así que desde el constructivismo se hizo referencia a las formas complejas en las que las personas aprenden mediante la reflexión, la gestión metacognitiva o de la propia actividad mental, el uso de sistemas simbólicos y dispositivos culturales, la cooperación interpersonal o el diálogo crítico con el conocimiento en diferentes contextos sociales formales e informales (Pozo, 2014).

Desde el constructivismo debe visualizarse que el conocimiento no es un fin en sí mismo, sino un medio imprescindible para que los alumnos sean competentes en un mundo donde el conocimiento académico es inabarcable, con duración y fiabilidad limitada, relativo desde la perspectiva adoptada y en constante transformación (Pozo, 2014; Pozo y Monereo, 2013; García, 2019).

Estas concepciones del aprendizaje se modificaron a partir de los modelos computacionales porque se concibió al aprendizaje desde la cognición y el procesamiento de la información; comenzaron a aplicarse principios como el aprendizaje asociativo conductista de tareas y escenarios más complejos como el aprendizaje de destrezas, lenguaje o la adquisición de reglas y conceptos que dieron lugar a un nuevo asociacionismo cognitivo en donde ya no se asocian estímulos y repuestas sino nodos semánticos, representaciones, pares condición-acción o incluso unidades de información subsimbólicas (Pozo, 2014). Por tanto, las suposiciones del aprendizaje se distanciaban del procesamiento de la información de forma conductista; se promovía asumir un compromiso de tipo constructivo donde se aplicara la reflexión consciente de los individuos sobre su propia acción que los dirija más a una metacognición y construcción consciente de su aprendizaje.

Es así como en la búsqueda de conceptualizaciones y explicaciones sobre cómo sucede el aprendizaje a partir de la irrupción de las tecnologías en el ámbito educativo, se incorporó al conectivismo como un referente para tratar de entender el aprendizaje en un contexto social emergente que se caracteriza por la creación de valor económico por medio de redes de inteligencia humana que crean conocimiento (Gutiérrez, 2012). En este sentido, George Siemens y Stephen Downes describen al conectivismo desde las limitaciones del conductismo, cognitivismo y constructivismo para explicar el efecto que la tecnología tiene sobre la forma como se vive en la actualidad, se aprende y se comunica (Gutiérrez, 2012; Duke, Harper y Johston, 2013).

Siemens acuñó el término conectivismo, el cual hacía referencia a la descripción de redes de aprendizaje. Su propuesta apunta al hecho de que el conocimiento se distribuye por medio de una red de conexiones (Downes, 2012) y, por lo tanto, aprender consiste en la capacidad de construir a través de esas redes, es decir, aprende quien es capaz de conectar los nodos que representan dichas redes. Este concepto hace mención a los procesos y espacios autoorganizados de aprendizaje que recogen la complejidad e informalidad de un proceso común en la construcción de conocimientos de un individuo.

Según Siemens (2004), el aprendizaje debe suceder a partir de un conjunto continuo de actitudes y acciones por parte de individuos y grupos que emplean la información para mantenerse al tanto de las situaciones conduciéndolos a aprender a lo largo de la vida y a construir nodos donde se concentra el conocimiento. Asimismo, Downes (2012) manifestó que aprender es un proceso de creación y eliminación de conexiones entre las entidades o el ajuste de las fortalezas de esas conexiones; en este sentido, se privilegia a las redes pero más allá del argumento electrónico. Lo que una red sabe no se encuentra en el contenido de sus entidades ni en el contenido de los mensajes que se envían entre ellas, sino que puede encontrarse mediante el reconocimiento de patrones emergentes en ésta o de conexiones e interacciones.

En este caso, las conexiones entre los nodos fortalecen el conocimiento que hay en cada uno de ellos al agrupar una serie de entidades que lo poseen y que gracias a su interacción y comunicación terminan convirtiéndola en algo 
con sentido y significado, aunque en palabras de Downes el término significado no le representa importancia; sin embargo, para los autores de este artículo, al aprender es necesario significar, porque finalmente el conocimiento se construye desde el significado y contexto de quien lo interpreta, apropia y expone.

Desde la postura de Siemens (2004), el conectivismo integra principios del caos, las redes y las teorías de la complejidad y autoorganización. El proceso de aprendizaje puede ocurrir en entornos confusos de elementos con volatilidad y que no están bajo el control de los individuos.

Se supone que el aprendizaje puede darse a partir de las conexiones que a la par permiten aprender más, y donde la interacción entre esas conexiones es más importante que el conocimiento mismo, toda vez que el conectivismo adquiere relevancia cuando hace notar que el conocimiento puede residir en espacios de almacenamiento exclusivos a los que acceden las personas adecuadas en el contexto adecuado para entonces aprehender de ello.

Si se parte de que la definición simple de conocimiento es la "facultad que tiene un ser humano para comprender por medio de la razón la naturaleza, cualidades y relaciones de las cosas" (Significados, 2020); de este modo, se diría que aprender desde la concepción del conectivismo es la capacidad que tiene una persona para conectar el conocimiento que reside en diferentes nodos y que comprende el resultado de la interacción entre éstos de acuerdo con la naturaleza, cualidades, contexto y preconcepciones que posee y modifica en función de cómo se adentra al entramado de conexiones que no necesariamente debe ser mecánica.

Desde la perspectiva de Downes (2012) -y tal como ya se expuso-, aprender es la creación y eliminación de conexiones entre las entidades o el ajuste de sus fortalezas; robustece su propuesta para ser considerada como una teoría porque desde su postura puede describirse cómo se crean o ajustan las conexiones implicando la similitud, contigüidad, retroalimentación y armonía.

Partiendo de esta premisa, aprender en la era digital implica ser parte de redes que se conectan bajo sentidos comunes, con nodos que comparten contenidos y conocimientos de acuerdo con un contexto o ámbito de interés, que cubren las necesidades de aprender de un individuo.

La operación de la red es continua: podrán integrarse nuevos nodos o desaparecer algunos, pero no se desestabilizan las interacciones que pueden producirse en ella; además, esa interacción va cargada de información que retroalimenta a los nodos para entrar en un proceso de construcción de aprendizajes, intercambio de conocimientos y producción de nuevas conexiones.

También, este proceso se realiza apoyado por las tecnologías de la información que adquieren el papel de depositarias de la información y conocimiento, así como de transportadoras, receptoras y expositoras.

En este mismo orden de ideas, las teorías pioneras en explicar el aprendizaje (conductismo, constructivismo, cognitivismo) por su propia naturaleza y tiempos de desarrollo no conciben el aprendizaje desde la incorporación de las Tecnologías de la Información y la Comunicación (TIC) (Cabero y Llorente, 2015). Con la aparición de las TIC comienza a facilitarse la creación de escenarios formativos en los que el uso de internet y sus aplicaciones es predominante y necesario, y en donde existen comunidades educativas formadas por profesores y alumnos o sólo alumnos o sólo profesores.

Asimismo, se supone mayor autonomía a los estudiantes. La comunicación que se privilegia es la asíncrona y la coincidencia de espacio y tiempo entre el profesor y el alumno ya no son tan necesarias. Bajo estas características, se da en los individuos un aprendizaje influenciado por el entorno, así como la adopción de formas de vida, actitudes, principios, aptitudes, etcétera, que les permiten estar inmersos en estas redes (Sánchez-Cabrera et al., 2019).

Estos escenarios de formación se caracterizan por poner al estudiante en el centro del proceso formativo: los sacan del trabajo aislado e individual para dirigirlo a posiciones sociales y colaborativas al tiempo que amplían los tipos de fuentes de información con los que se puede interactuar.

En la actualidad el aprendizaje requiere que el estudiante ponga en acción diferentes competencias para pensar sobre conexiones, resolver problemas, enfrentar retos, pensar conceptos, criticar y evaluar, crear y compartir conocimiento, entre otras habilidades que las teorías clásicas del aprendizaje no vislumbraron en su momento con la mediación de las tecnologías (Cabero y Llorente, 2015). 
A partir de estos supuestos, Sánchez-Cabrera et al. (2019), Cabero y Llorente (2015) coinciden en que en esta época el conocimiento no es un objeto que se consigue o se compre. Por el contrario, tiene un significado a partir de la experiencia, de lo que se ha encontrado o conectado entre nosotros mismos; por tanto, el conocimiento no se relaciona con un producto; no se cosifica si no tiene capacidad de conexión y asociación con lo que ya se sabe.

A pesar de lo mencionado, Duke, Harper y Johston (2013) afirman que existen ciertas restricciones para considerar a un cúmulo de conocimientos como una teoría base que defina al aprendizaje. Para ello es necesario partir de pruebas y observaciones que manifiesten: a) la predisposición que existe de un individuo a aprender, b) cómo están diseñados los conceptos que facilitarán su comprensión y c) cómo se ha dado seguimiento a las ideas que representan el cuerpo de conocimiento en que se sustentan. Sólo atendiendo a estos supuestos puede comprenderse por qué para algunos autores en la actualidad el conectivismo es considerado una teoría, mientras que para otros no lo es.

En este sentido, la postura de los autores de este artículo es considerar al conectivismo como una disciplina que se acerca a describir procesos que suceden cuando se aprende en ambientes mediatizados por las tecnologías.

En este caso, el conocimiento se intercambia y, a su vez, es producido por los propios individuos debido a la naturaleza de su ubicuidad y al acceso que se tiene a nubes de conexiones cargadas de contenidos que se pueden relacionar y les permiten construir algo nuevo a partir de los vínculos que establecen entre lo que están conociendo y lo que ya sabían.

Por tanto, la pregunta plateada en el título de este apartado sigue sin una respuesta concreta. El conectivismo puede considerarse una moda teniendo en cuenta la novedad que imprimen las tecnologías en nuestra sociedad, y puede ser una teoría si a partir de ciertas visiones éste describe, en función de sus principios, cómo un individuo aprende en una sociedad en red y ser una disciplina en la medida que hace emerger elementos que sustentan el proceso de comunicación, la interacción y la relación entre nodos, las tecnologías y los individuos.

Sin embargo, para evitar esta confusión, y fijar una postura, para quien aquí escribe, el conectivismo se concibe como disciplina puesto que se concibe como un campo de conocimiento vigente, del que se está hablando y produciendo documentación y conocimiento en la medida en que se reportan investigaciones que sustentan su aplicabilidad en los procesos formativos.

\section{Neuroeducación, ¿La panacea para la MeJora educativa?}

La neuroeducación es una disciplina que se refiere a los estudios del cerebro y los procesos de aprendizaje que aporta información a la enseñanza (Pallarés-Domínguez, 2016). En este sentido, la finalidad de dicha disciplina es obtener mejores resultados en la adquisición, retención y aplicación del aprendizaje en el educando (Pherez, Vargas y Jerez, 2018; Ramón, 2015; Caicedo, 2016).

La idea de la neuroeducación es que con la ayuda de diversas estrategias puedan generarse nuevas formas de enseñanza que produzcan modelos pedagógicos coincidentes con el desarrollo del cerebro en las diferentes etapas de vida de una persona, por ejemplo: que quien genera ambientes de formación conozca las maneras en que se aprende, qué motiva al aprendizaje, qué es lo que más se valora y cómo se retiene y utiliza la información que se recibe, etcétera. Conocer el funcionamiento del cerebro por parte de quien enseña promueve la generación de contextos educativos a los que los estudiantes se adaptan a través de su comportamiento y los posibilita para poder transformarlos si así se requiere (Bueno, 2017; Ramón, 2015).

A decir de Pallarés-Domínguez (2016), Pherez, Vargas y Jerez, (2018) y Béjar, (2014), la neuroeducación se concibe como una ciencia cognitiva aplicada, donde el principal objetivo es destacar el papel que tiene el estudio del cerebro en beneficio de la educación, puesto que podría aprovecharse para generar metodologías, revisar presupuestos o estudiar trastornos del proceso de enseñanza-aprendizaje. Sin embargo, es necesario cuidar el hecho de no caer en mitos que en lugar de beneficiarla podrían perjudicarla; es el caso de las personas que aprenden mejor cuando reciben información en su estilo de aprendizaje preferido o, cómo se explican las diferencias entre 
individuos, a partir del dominio de los hemisferios cerebrales (Blanchette y Masson, 2015; Gracia, 2018; Guillén y Forés, 2017).

El concepto de neuroeducación sigue en controversia, puesto que la postura de quienes lo estudian tiene cierto tinte de escepticismo cuando se hace referencia a que, basándose en esta disciplina, se pueden modificar las conductas de los aprendices y, más aún, las estructuras cerebrales. Bowers (2016) argumenta, por ejemplo, que no existe información actualizada que confirme que la neuroeducación se base en métodos de enseñanza nuevos y efectivos y pone en duda que el conocimiento del cerebro sea suficiente para caracterizar las capacidades cognitivas de los estudiantes cuando éstas pueden observarse desde los comportamientos que ellos tienen. Por este motivo, sugiere que debe existir una estrecha comunicación entre neurocientíficos y educadores para encontrar las respuestas que aún no son obvias o que están en el tintero de los investigadores.

En este sentido, Geake (2008) y Varas-Geneistier y Ferreira (2017) apoyan la postura de que los estilos de aprendizaje y la dominación de los hemisferios cerebrales no son indicadores suficientes para llevar a cabo prácticas educativas mejoradas, ya que no existe una validación científica suficiente sobre la efectividad de experiencias basadas en estos argumentos. Por ello, estos autores recomiendan que la educación fundada en el estudio del cerebro se cimente en evidencias científicas que hayan comprobado lo anterior. Es decir, el currículum escolar puede estructurarse ofreciendo múltiples oportunidades de aprendizaje que promuevan las habilidades cognitivas de los individuos en función de sus capacidades, siempre y cuando no se dejen influenciar por información no comprobada o que puede resultar de fuentes no confiables.

Bajo este orden de ideas, dar respuesta de cómo suceden los procesos de aprendizaje en los individuos es un reto que la educación aún tiene pendiente de resolver. La innovación exigida por la revolución de la sociedad en la que nos desarrollamos implica transformar las enraizadas tradiciones de la enseñanza. En este sentido, las metodologías de enseñanza-aprendizaje adoptadas en los diferentes niveles educativos han dado cabida a los diálogos interdisciplinarios entre las ciencias cognitivas y la neurociencia, acción que parece gestar un verdadero cambio (Pherez, Vargas y Jerez, 2018).

Por otra parte, hay quien argumenta que las neurociencias están aportando elementos de transformación a los paradigmas de la educación. Con base en la neurodidáctica, por ejemplo, se analizan las competencias que tiene el cerebro y se llega a la comprensión de la diversidad personal en el proceso de aprendizaje (Paniagua, 2013). La unión de las ciencias cognitivas y las neurociencias con la educación permitiría diseñar estrategias didácticas y metodológicas eficientes con el fin de promover un mayor aprendizaje a partir de la capacidad que tengan los profesores de interpretarlo.

Sin duda, debería considerarse a la neurodidáctica como una temática educativa que requiere mayor presencia en los procesos de formación docente, puesto que su comprensión ayudaría a que la práctica de los profesores impulsara procesos innovadores, creativos, críticos y hasta propositivos, toda vez que al conocer el funcionamiento del cerebro, el docente puede incidir en el proceso de aprendizaje del estudiante y lo motive a ser autónomo, independiente y autorregulado (Pherez, Vargas, y Jerez, 2018).

En este orden de ideas, la neuroeducación representa la respuesta científica que los educadores tendrían que considerar acerca de lo que debe hacerse de nuevo en clase con los alumnos (Béjar, 2014). Por ello, esta disciplina intenta usar la información que se revela en las neuroimágenes y que pueden aportar datos que ayuden a la comprensión de la interacción del cerebro con el entorno en cada proceso de enseñanza-aprendizaje en donde participa un individuo y pone en juego sus habilidades cognitivas (Codina, 2014). Por tanto, esta naciente disciplina trata de armonizar las metodologías de enseñanza de los profesores con las técnicas de aprendizaje de los estudiantes.

Aunado a lo anterior, debe considerarse que las emociones de los individuos juegan un papel primordial en el proceso de enseñanza-aprendizaje. Si no existe motivación por parte de ambos actores (docente-estudiante), será complejo lograr capacidad para prestar atención y, por supuesto, para aprender. Tal y como sostienen Pherez, Vargas y Jerez (2018), cuando los sentimientos son ignorados se puede sabotear el aprendizaje, la atención y la memoria dejando de lado el diseño de estrategias didácticas y metodológicas que promuevan un mayor desarrollo 
cerebral o de aprendizaje. Es conocido que en la actualidad pueden encontrarse estudiantes desmotivados con pocas o nulas ganas de aprender, sin iniciativa e interés, por lo que es necesario realizar distintas intervenciones para modificar esa situación y lograr revertir la actitud de los aprendices (Muchiut et al., 2018).

Para conseguir este panorma es necesario que los docentes consideren tres momentos formativos indispensables en su práctica: diseñar su acción pedagógica, implementarla, establecer cómo finalizará y cómo será evaluada; de esta manera, se tendrá una visión integral que puede incluir aspectos neuroeducativos que abonarían en la mejora de su ejercicio (Guillén y Forés, 2017). De igual manera, Guillen y Forés (2017) proponen que el docente debe diseñar pedagógicamente su acción educativa para lograr que el alumno se sienta seguro y participe de forma activa en su aprendizaje con seguridad y expectativas claras de lo que quiere alcanzar, además de proyectar un crecimiento en su comprensión y adaptación al ambiente de aprendizaje en el que se desempeña, y abonar así en la construcción de conocimientos desde su motivación.

Si el docente lograra esto, sería benéfico, puesto que su rol cambiaría al estimular el desarrollo de los estudiantes en virtud de unas prácticas de enseñanza fundamentadas en el conocimiento del cerebro.

Los conocimientos en neuroeducación, por tanto, pueden contribuir en la generación de entornos adecuados y enriquecidos donde despierte el cerebro para el aprendizaje, ya que éste aprende desde diferentes vías y tiene la capacidad de establecer rutas gradualmente. Por ello, las propuestas de aprendizaje deben considerarse partiendo de lo simple y concreto a lo más abstracto y complejo (Pherez, Vargas y Jerez, 2018).

\section{Los RESUltados de la FASE HeUrística}

En este apartado se muestran en números los resultados de la fase heurística del artículo. Se cuidó que la mayor parte de las referencias correspondieran a la última década e inclusive se dio mayor prioridad a la literatura del último quinquenio.

Ante la búsqueda de información para realizar este trabajo, se encontraron un total de 100 referencias, las cuales cumplieron con los criterios de búsqueda: fecha de publicación, tipo, idoma, información que aporta y si fue resultado de una investigación ( 46 abordan de distintas formas al conectivismo y 54 a la neuroeducación). De la totalidad de dichos documentos, 82 corresponden a artículos de revistas indexadas alojadas en diversas bases de datos, 3 son tesis de maestría y 14 son libros de texto, 71 están escritos en español y 22 en inglés; también se identificó que 77 corresponden a recopilaciones documentales que hacen descripción de las disciplinas sin llegar a explicar una metodología o contar con datos empíricos que los sustenten, por lo que sólo se basan en supuestos o recopilaciones de literatura producida por otros autores; además, 8 representan estudios con enfoque cuantitativo, 10 cualitativos y 3 reseñan investigación aplicada sin mencionar el enfoque, alcance o resultados obtenidos.

La información anterior refuerza el supuesto de quien aquí escribe respecto a que la producción científica que abona al campo de conocimiento de estas disciplinas es incipiente y no cuenta con suficientemente soporte en investigaciones que comprueben su impacto o den cuenta de su incidencia en el proceso de enseñanza-aprendizaje, de tal forma que lo comunicado hasta la fecha se basa gran parte en los qué y cómo debe llevarse a cabo una práctica formativa; sin embargo, poco se señalan los impactos identificados. Cabe mencionar que ninguna referencia de las que se colectaron para este trabajo habla de una combinación del conectivismo y neuroeducación como fundamento de experiencias de enseñanza. Así pues, esta deficiencia se convierte en un área de oportunidad para gestar investigaciones en las que ambas disciplinas se tomen como cimiento de prácticas docentes y de ambientes de aprendizaje innovadores, mientras que las exigencias de la sociedad del conocimiento en la cual nos desarrollamos reclama adaptación, renovación o transformación de prácticas arcaicas o basadas en sustentos del suceder del aprendizaje cuando no se contempló la aparición de las TIC como mediadoras del proceso y que, por ende, han acarreado una modificación en las habilidades cognitivas simples y de orden superior que impactan directamente en el funcionamiento del cerebro de estudiantes y docentes. 


\section{FASE hermenéutica, la inTerPretación de los hallazgos}

A partir de la revisión, se planteó el siguiente método la interpretación de los hallazgos:

a) Identificar y recolectar documentos útiles para lograr el objetivo del artículo

b) Definir las categorías de análisis

c) Realizar un análisis en función de las categorías y plasmarlo desde la interpretación propia

d) Identificar los elementos de cada disciplina que pueden ser comunes y aplicarse de forma vinculada

Las categorías establecidas fueron cuatro:

1. Postura ante las disciplinas

2. Habilidades cognitivas que promueve

3. Descriptores aplicativos que permiten vincularlas

4. Rol de los actores implicados según los aportes de la disciplina.

La tabla 1 presenta el concentrado de información referido a estas categorías.

A partir de lo anterior, es necesario que las instituciones educativas asuman posturas con la capacidad de promover la creación e implementación de modelos didácticos y pedagógicos que compartan teorías de enseñanzaaprendizaje renovadas, y las cuales estén fundamentadas en las necesidades que exige la evolución en donde el sistema educativo se encuentra inmerso.

$\mathrm{Al}$ respecto, debe tomarse conciencia de que transferir información ya no es suficiente, puesto que puede obtenerse en internet desde diversos dispositivos. Lo importante ahora es que el docente ejerza un papel de guía para que los estudiantes desarrollen criterios de búsqueda y de aprendizaje de contenidos y sean capaces de adquirir la información, integrarla y procesarla desde una postura creativa, crítica e intuitiva. La figura 1 muestra la forma en cómo se visualiza que ambas disciplinas se integren para sustentar la premisa de la que se partió para este artículo.

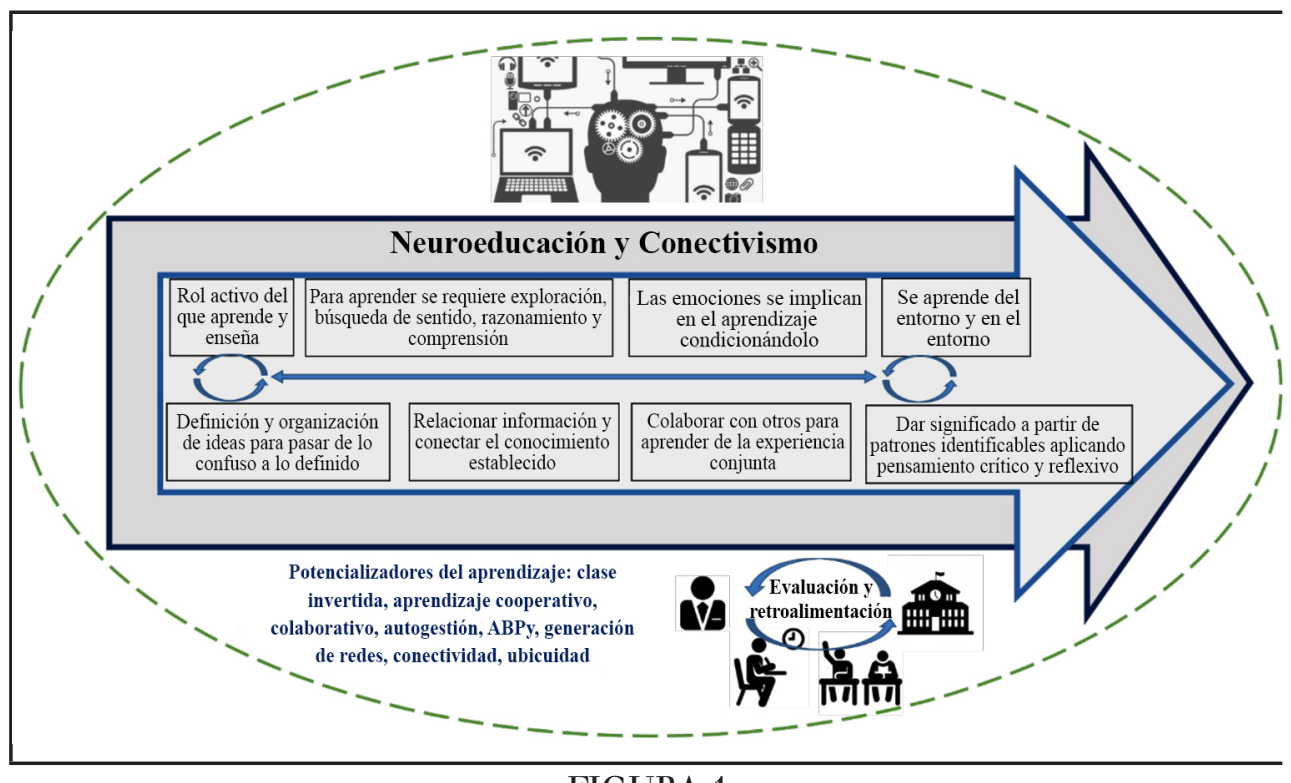

\section{FIGURA 1}

Integración de neuroeducación y conectivismo

Fuente: elaboración propia. 
TABLA 1

Análisis de información plasmado desde la interpretación propia en función de las categorías establecidas

\begin{tabular}{|c|c|c|}
\hline $\begin{array}{l}\text { Categorías de } \\
\text { análisis }\end{array}$ & Conectivismo & Neuroeducación \\
\hline $\begin{array}{l}\text { 1. Postura ante las } \\
\text { disciplinas }\end{array}$ & $\begin{array}{l}\text { Escepticismo y reserva para considerarse una } \\
\text { teoría porque no parte de elementos empíricos } \\
\text { suficientes ni tiene un campo de estudios } \\
\text { amplio como las teorías del aprendizaje que } \\
\text { le anteceden. } \\
\text { El conectivismo tiende a explicar la calidad } \\
\text { del acceso al conocimiento distribuido, } \\
\text { pero no se enfoca precisamente en el cómo } \\
\text { se aprende, puesto que se fundamenta en } \\
\text { el supuesto de que éste puede residir en } \\
\text { artefactos no humanos cuando los estudiosos } \\
\text { del aprendizaje aseguran que ocurre sólo } \\
\text { dentro de una persona. } \\
\text { Desde esta disciplina se concibe al aprendizaje } \\
\text { como algo continuo y que dura para toda } \\
\text { la vida, ya que el individuo desarrolla la } \\
\text { habilidad cognitiva del procesamiento de } \\
\text { información comprendiendo dónde puede } \\
\text { encontrar el conocimiento necesario. } \\
\text { Considera el actual proceder de los individuos } \\
\text { que se adaptan a una realidad social en la } \\
\text { que hay conexiones de forma ubicua que los } \\
\text { lleva a compartir y construir conocimiento } \\
\text { a partir de la interacción y relaciones que se } \\
\text { establecen. } \\
\text { Se aprende cuando la conexión genera } \\
\text { esquemas mentales a través de dichas } \\
\text { conexiones; en este sentido, se construye un } \\
\text { entramado de redes que son actualizables } \\
\text { y que no necesariamente requieren que el } \\
\text { conocimiento esté depositado en la memoria } \\
\text { de los individuos, sino apoyado por los } \\
\text { dispositivos y aplicaciones tecnológicas que } \\
\text { fungen como nodos vivos en función de las } \\
\text { necesidades de comprensión y esquemas que } \\
\text { las implica. }\end{array}$ & $\begin{array}{l}\text { Aunque promueve una mejora y hasta cambio de la } \\
\text { educación actual también puede ser una panacea, } \\
\text { por lo que se considera que debe tomarse como } \\
\text { fundamento con cautela y reserva. } \\
\text { La neuroeducación está dirigida a explicar la } \\
\text { organización y funcionamiento del cerebro } \\
\text { cuando sucede el aprendizaje en un individuo, } \\
\text { por lo cual busca los elementos necesarios para } \\
\text { atender las características personales y enfocarse } \\
\text { mientras tanto a la diversidad en función de las } \\
\text { características únicas. } \\
\text { Esta disciplina se considera una rama de } \\
\text { la pedagogía, la cual busca otorgar nuevas } \\
\text { orientaciones para el diseño de estrategias } \\
\text { didácticas y metodológicas más eficientes que } \\
\text { promuevan un mayor desarrollo cerebral a mayor } \\
\text { aprendizaje. } \\
\text { A esta disciplina se le considera como ciencia } \\
\text { moderna porque estudia el cerebro y puede ser } \\
\text { un referente para explicar procesos físicos y } \\
\text { psíquicos, además es transdisciplinar y abarca a } \\
\text { la neurociencia cognitiva, las ciencias cognitivas, } \\
\text { la medicina y la educación. } \\
\text { En esta disciplina es recomendable observar la } \\
\text { conducta de los aprendices, pues de ahí se puede } \\
\text { obtener más información respecto al aprendizaje } \\
\text { y cómo se lleva a cabo sin limitar únicamente a } \\
\text { las imágenes producto de resonancias. Promueve } \\
\text { el conocimiento del cerebro en función de la } \\
\text { proacción que exigen los cambios vertiginosos } \\
\text { de la sociedad. }\end{array}$ \\
\hline $\begin{array}{l}\text { 2. Habilidades } \\
\text { cognitivas que } \\
\text { promueve }\end{array}$ & $\begin{array}{l}\text { - Creatividad } \\
\text { - Pensamiento crítico } \\
\text { - Selección y discriminación de contenidos } \\
\text { - Toma de decisiones } \\
\text { - Autodisciplina } \\
\text { - Organización de actividades basadas en } \\
\text { experiencias auténticas } \\
\text { - Metacognición } \\
\text { - Aprendizaje cooperativo } \\
\text { - Interacción independiente del tiempo y el } \\
\text { espacio }\end{array}$ & $\begin{array}{l}\text { - Reconocimiento de esquemas en conjunto de } \\
\text { eventos neuronales } \\
\text { - Pensamiento crítico } \\
\text { - Habilidades cognitivas superiores } \\
\text { - Metacognición } \\
\text { - Inteligencia emocional } \\
\text { - Desarrollo del lenguaje } \\
\text { - Significación del aprendizaje } \\
\text { - Autonomía } \\
\text { - Independencia } \\
\text { - Pensamiento creativo }\end{array}$ \\
\hline
\end{tabular}


TABLA 1 (continúa)

\begin{tabular}{|c|c|}
\hline $\begin{array}{l}\text { Categorías de } \\
\text { análisis }\end{array}$ & Neuroeducación \\
\hline $\begin{array}{l}\text { 3. Descriptores } \\
\text { aplicativos } \\
\text { que permiten } \\
\text { vincularlas }\end{array}$ & $\begin{array}{l}\text { - Resolución de problemas } \\
\text { - Enfocar el aprendizaje en el estudiante } \\
\text { - Organizar el aprendizaje de acuerdo con los intereses, aptitudes y diferencias individuales de } \\
\text { los estudiantes } \\
\text { - Promover situaciones donde los docentes y estudiantes aprendan juntos } \\
\text { - Movilizar la creatividad para resolver problemas y generar contenidos acordes con las demandas } \\
\text { educativas } \\
\text { - Generar la capacidad de autogestión en el aprendizaje } \\
\text { - Promover la comprensión y significación de los contenidos que se aprenden por diversos medios } \\
\text { - Motivar al aprendizaje cooperativo } \\
\text { - Promover el pensamiento crítico y creativo } \\
\text { - Aplicar la metacognición }\end{array}$ \\
\hline $\begin{array}{l}\text { 4. Rol de los } \\
\text { actores }\end{array}$ & 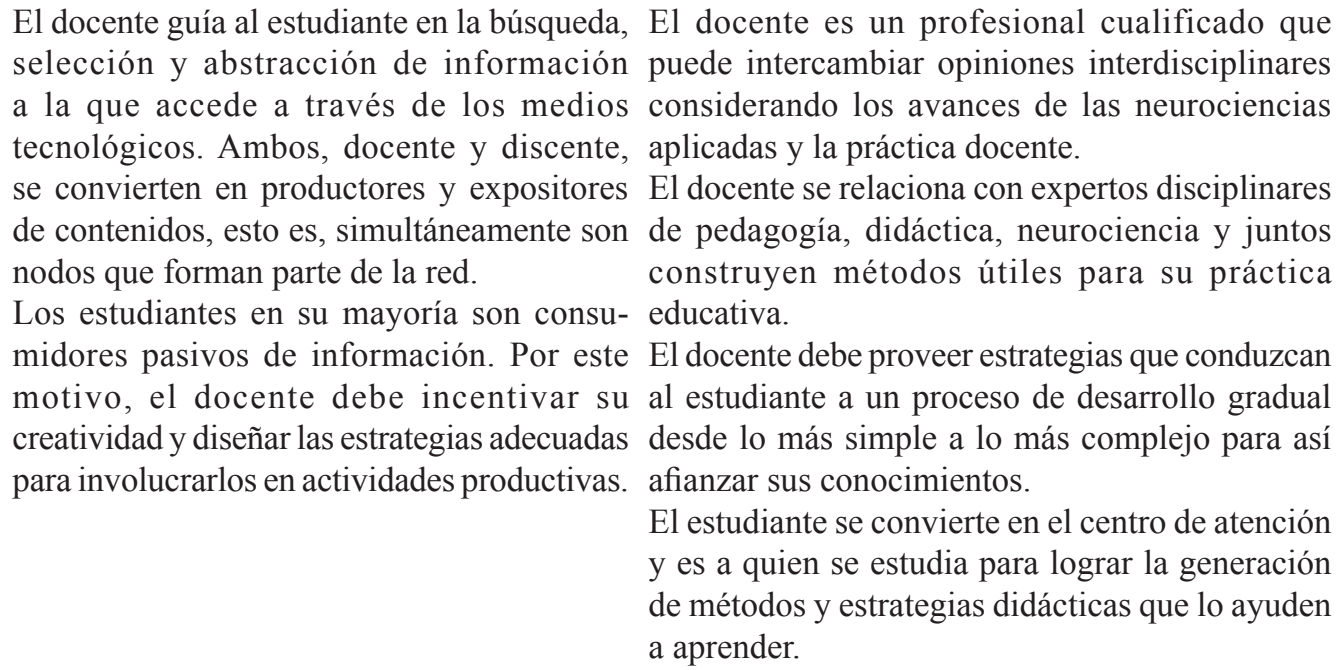 \\
\hline
\end{tabular}

Como puede observarse, la integración de ambas disciplinas se concibe desde la unión de principios neuroeducativos y del conectivismo, puesto que no se contraponen, sino que complementan los procesos de enseñanza-aprendizaje en donde el docente y estudiante juegan roles de intercambio, aprendan el uno del otro, lo cual implicaría también a la institución educativa, ya que desde sus políticas y criterios podrán implementarse estas transdisciplinas para la formación en la era digital.

Desde la postura de este artículo se les ha denominado transdisciplinas porque no son aplicativas o exclusivas de una sola área del conocimiento o etapa de formación; al contrario, por su naturaleza se implican en cualquier proceso de enseñanza o aprendizaje y a distintos niveles educativos. En resumen, se requiere de alumnos y profesores dispuestos a ponerlas en práctica y hacerse conscientes de que a pesar de su incipiente expansión aportan elementos que adaptan los procesos formativos actuales a las necesidades que el entorno exige.

\section{Conclusiones y PRospectiva}

A partir de la revisión de literatura colectada para este artículo y del objetivo propuesto, se ha expuesto crítica y analíticamente cómo el conectivismo y la neuroeducación son transdisciplinas que explican procesos formativos en la era digital. También, pudo constatarse que ambas se mantienen en el escepticismo de diversos autores, o en la aceptación de otros. Desde la postura de quien aquí escribe, dichas disciplinas pueden fortalecer los procesos 
de enseñanza-aprendizaje en el sentido de conocer lo que internamente motiva a un individuo a aprender a partir del conocimiento del cerebro y esto por consecuencia da información valiosa para diseñar o estructurar técnicas, estrategias y metodologías que abonen a la mejora y adaptación de las necesidades de los estudiantes en los ambientes educativos emergentes, donde la ubicuidad se hace aún más notoria. Ambas disciplinas dan posibilidades de autonomía a los estudiantes, ya que aprenden por ellos mismos en función de lo que necesitan conocer, ya sea por gusto u obligación. De esta manera los alumnos aprenden a afrontar retos a su propio ritmo porque el docente los guía para conseguirlo.

Si el profesor sabe dirigirlos en términos de lo que conoce e identifica de ellos, logrará que cada estudiante alcance resultados desde su libertad, creatividad y capacidad para resolver problemas o tomar decisiones y cumpla con los objetivos de aprendizaje que les haya indicado.

Por lo tanto, se esperaría que el profesor gracias a sus prácticas de enseñanza logre motivar a los estudiantes a que exploren la infinidad de posibilidades y habilidades que pueden desarrollar para solucionar problemas, tomar decisiones o afrontar retos de acuerdo con sus propias capacidades y no desde la generalidad u homogeneización como se acostumbra en la enseñanza tradicional. Esto, además, potencializa el aprender de y con los demás, pues al vivir las experiencias individuales y compartirlas con otros los ayuda a construir esos nodos que, mediados por las tecnologías, les permiten crear conocimiento desde la conectividad y colectividad y acercarse así a lo que pretende explicar el conectivismo respecto al aprendizaje en la era digital.

Para ambas disciplinas la significación tiene gran importancia porque permite tanto a docentes como a discentes profundizar en sus habilidades personales, actitudes y aptitudes que, en consecuencia, les faciliten el proceso de enseñar o aprender.

De esta manera, descubrir los patrones ocultos que existen, a partir de las capacidades desarrolladas, los acercan a la comprensión de la realidad desde una visión integral que implica a las TIC como mediadoras.

Cabe mencionar que estas disciplinas también pueden ayudar a reducir las brechas generacionales entre los inmigrantes y los nativos digitales, puesto que por las características de las épocas en las que nacieron y se educaron sus cerebros, formas de aprender, comunicarse, cooperar, colaborar y producir son distintas; sin embargo, llega el momento en que cohabitan cuando los procesos formativos los unen en un ambiente donde deben adaptarse los unos a los otros para lograr construir conocimientos que sean útiles en el contexto donde se desempeñan.

Finalmente, para consolidar la información que se ha tomado de la literatura o que en su caso se ha expresado en este trabajo, es necesario que se realicen investigaciones con enfoques epistémicos que arrojen información empírica que fortalezca las premisas de las que parte cada una de las disciplinas y que también se corrobore que ambas pueden coexistir y mejorar las prácticas educativas.

\section{REFERENCIAS}

Béjar, M. (2014). Una mirada sobre la educación, neuroeducación. Padres y Maestros (355), 49-52. Disponible en https://revistas.comillas.edu/index.php/padresymaestros/article/view/2622

Blanchette, J. y Masson, S. (2015). EdCanNetwork. Disponible en https://www.edcan.ca/articles/neuromythsin-education/

Bowers, J. (2016). The practical and principled problems with educational neuroscience. Psychological Review, 123. https://doi.org/600-612. doi:10.1037/rev0000025

Bueno, D. (2017). Neurociencia para educadores. Barcelona: Octaedro.

Cabero, J. y Llorente, M. (2015). Tecnologías de la Información y la Comunicación (TIC): escenarios informativos y teorías del aprendizaje. Revista Lasallista de Investigación, 12(2), 186-193. 
Caicedo, H. (2016). Neuroeducación. Una propuesta educativa en el aula de clase. México: Ediciones de la U.

Codina, M. J. (2014). Neuroeducación: reflexiones sobre neurociencia, filosofía y educación. Postconvencionales, 7(8), 164-181. Disponible en https://dialnet.unirioja.es/servlet/ articulo?codigo $=4807471$

Czerwonogora, A. (2014). El aprendizaje en la era digital: nuevos escenarios para el mundo conectado, en E. Fiore y J. Leymonié, Didáctica práctica para enseñanza básica, media y superior (pp. 235-257). Montevideo: Magro.

Downes, S. (2012). Connectivism and connective knowledge. Canada: CC by NC-SA.

Duke, B., Harper, G., \& Johston, M. (2013). Connectivism as a digital age learning. The International HETL Review, 4-13. Retrieved from https://www.hetl.org/wp-content/uploads/2013/09/ HETLReview2013SpecialIssueArticle1.pdf

García, E. (2019). Cómo desaprender y reaprender: el cerebro no se formatea pero podemos hacerlo. Disponible en https://aunclicdelastic.blogthinkbig.com/desaprender-y-reaprender-en-la-era-digital-podemoshacerlo

Geake, J. (2008). Neuromythologies in education. Educational Research, 50(2), 123-133. https://doi. org/10.1080/00131880802082518

Gracia, J. (2018). El fin ético no naturalista de la neuroeducación. Revista de pensament I Análisi (22), 51-68. https://doi.org/10.6035/Recerca.2018.22.4

Guerrero, J. (2019). Docentes del dia. Disponible en https://docentesaldia.com/2019/02/02/ensenara-pensar-30-preguntas-para-promover-la-metacognicion-en-el-aula/

Guillén, J. C. y Forés, A. (2017). ¿Qué nos dice la neuroeducación acerca de las pedagogías emergentes? En A. Forés y E. Subias, Pedagogías emergentes: 14 preguntas para el debate (pp. 67-87). Barcelona: Octaedro.

Gutiérrez, L. (2012). Conectivismo como teoría de aprendizaje: conceptos, ideas y posibles limitaciones. Revista Educación y Tecnología, 1, 111-122.

Muchiut, Á. F., Zapata, R. B., Comba, A., Torres, N., Mari, M., Pellizardi, J., y Segovia, A. P. (2018). Neurodidáctica y autorregulación del aprendizaje, un camino de la teoría a la práctica. Revista Ibero-Americana de Educación, 78(1), 183-204.

Padrón, G. (2014). Critica al conectivismo. Disponible en https://es.scribd.com/document/293364427/ Critica-Al-Conectivismo-o-Conectismo

Pallarés-Domínguez, D. (2016). Neuroeducación en diálogo: Neuromitos en el proceso de enseñanzaaprendizaje y en la educación moral. Pensamiento, 72(273), 941-958. https://doi.org/pen.v72. i273.y2016.010

Paniagua, M. N. (2013). Neurodidáctica: una nueva forma de hacer educación. Fides et Ratio, 72-77. Disponible en http://www.scielo.org.bo/pdf/rfer/v6n6/v6n6_a09.pdf

Pherez, G., Vargas, S. y Jerez, J. (2018). Neuroaprendizaje, una propuesta educativa: herramientas para mejorar la praxis del docente. Civilizar Ciencias Sociales y Humanas, 18(34). https://doi. org/10.22518/usergioa/jour/ccsh/2018.1/a10

Pozo, J. I. (2014). Psicologia del aprendizaje humano. Madrid: Morata.

Pozo, J. I. y Monereo, C. (2013). La nueva cultura del aprendizaje universitario o por qué cambiar nuestras formas de enseñar y aprender, en J. I. Pozo y M. D. Pérez, Psicología del aprendizaje universitario: la formación por competencias (pp. 9-28). Madrid: Morata. 
Ramón, M. (2015). Neuroeducación un desafío para los docentes. Disponible en https://intef.es/Blog/ neuroeducacion-un-desafio-para-los-docentes/

Sagar, C. (2014). El conectivismo, o aprender en nubes de conexiones. Educación y Comunicación, 9, 137-148. Disponible en https://www.academia.edu/12459347/El_conectivismo_o_aprender_en_nubes_de_ conexiones

Sánchez-Cabrera, R., Costa-Román, Ó., Mañoso-Pacheco, L., Novillo-López, M. y Pericacho-Gómez, F. (2019). Orígenes del conectivismo como nuevo paradigma del aprendizaje en la era digital. Educación y Humanismo, 21(36), 121-142. https://doi.org/dx10.17081/eduhum.21.36.3265

Siemens, G. (2005).www.comenius.cl. Disponible en https://www.comenius.cl/recursos/virtual/minsal_v2/ Modulo_1/Recursos/Lectura/conectivismo_Siemens.pdf

Significados. (2020). Conocimiento. Disponible en https://www.significados.com/conocimiento/

Sitti, S., Sopeerak, S., \& Sompong, N. (2013). Development of instructional model based on connectivism learning theory to enhance problem-solving skill in ICT for Daily life of higher education etudents. Procedia. Social and behavioral Sciences, 315-322. https://doi.org/ 10.1016/j.sbspro.2013.10.339

Varas-Geneistier, P. y Ferreira, R. A. (2017). Neuromitos de los profesores chilenos: orígenes y predictores. Estudiospedagógicos, 43(3), 341. Disponible en http://www.redalyc.org/pdf/1735/173554750020.pdf

\section{BY-NC-ND}

